\title{
Radiotherapy of prostate cancer: impact of treatment characteristics on the incidence of second tumors
}

\author{
Milly BUWENGE ${ }^{1 *}$ D, Erica SCIROCCO ${ }^{1}$, Francesco DEODATO², Gabriella MACCHIA ${ }^{2}$, Maria NTRETA', Silvia BISELLO', \\ Giambattista SIEPE ${ }^{1}$, Savino CILLA ${ }^{3}$, Anna Rita ALITTO ${ }^{4}$, Vincenzo VALENTINI ${ }^{4,5}$, Lidia STRIGARI ${ }^{6}$, \\ Alessio G. MORGANTI ${ }^{1+}$ and Silvia CAMMELLI ${ }^{1+}$
}

\begin{abstract}
Background: It has been hypothesized that radiotherapy (RT) techniques delivering radiations to larger volumes (IMRT, VMAT) are potentially associated with a higher risk of second primary tumors. The aim of this study was to analyse the impact of RT technique (3D-CRT vs IMRTNMAT) on the incidence of second tumors in prostate cancer (PCa) patients.

Methods: A retrospective study on 2526 previously irradiated PCa patients was performed. Patients were treated with 3D-CRT (21.3\%), IMRT (68.1\%), or VMAT (10.6\%). Second tumors incidence was analysed in 3 categories: pelvic, pelvic and abdominal, and "any site". The correlation with RT technique was analysed using log-rank test and Cox's proportional hazard method.

Results: With a median follow-up of 72 months (range: 9-185), 92 (3.6\%) cases of second tumors were recorded with 48 months (range: 9-152) median interval from RT. Actuarial 10-year second tumor free survival (STFS) was 87.3\%. Ten-year STFS in patients treated with 3D-CRT and IMRTNMAT was 85.8 and 84.5\%, respectively (p: .627). A significantly higher 10-year cumulative incidence of second tumors in the pelvis was registered in patients treated with IMRTNMAT compared to 3D-CRT (10.7\% vs 6.0\%; $p:$.033). The lower incidence of second pelvic cancers in patients treated with 3D-CRT was confirmed at multivariable analysis (HR: 2.42, 95\%Cl: 1.07-5.47, p: .034).
\end{abstract}

Conclusions: The incidence of second pelvic tumors after RT of PCa showed a significant correlation with treatment technique. Further analyses in larger series with prolonged follow-up are needed to confirm these results.

Keywords: Second malignancy, 3D-conformal radiotherapy, Intensity modulated radiotherapy, Volumetric modulated arc therapy, Prostate neoplasms

\section{Background}

Prostate cancer $(\mathrm{PCa})$ is the second most common cancer in men worldwide [1]. In the USA, data from the Surveillance, Epidemiology and End Results database led to a forecast of approximately 174,650 new diagnoses and 31,620 deaths from PCa in 2019 [2].

Radiotherapy (RT) has been used in the treatment of PCa for over 70 years. RT results have gradually improved

\footnotetext{
* Correspondence: mbuwenge@gmail.com

${ }^{\dagger}$ Alessio G. MORGANTI and Silvia CAMMELLI contributed equally to this work.

${ }^{1}$ Radiation Oncology Center, Department of Experimental, Diagnostic and

Specialty Medicine - DIMES, University of Bologna, S. Orsola-Malpighi

Hospital, via Giuseppe Massarenti 9, 40138 Bologna, Italy

Full list of author information is available at the end of the article
}

over time thanks to the technological evolution and to the combination with adjuvant androgen deprivation therapy (ADT). However, some studies suggested that patients undergoing RT show a slightly higher incidence of second primary tumors particularly in the pelvis [3-5], although other authors attributed this increased risk to other factors such as age and lifestyle [6].

In the late 1990s, 3-dimensional conformal RT (3DCRT) emerged as the optimal RT technique for this tumor due to improved dose distribution compared to conventional 2-dimensional RT. In fact, a significant reduction of acute and late toxicity was demonstrated [7, 8]. In the following decade, 3D-CRT was progressively

(c) The Author(s). 2020 Open Access This article is distributed under the terms of the Creative Commons Attribution 4.0 International License (http://creativecommons.org/licenses/by/4.0/), which permits unrestricted use, distribution, and 
replaced in this setting by modulated RT techniques such as intensity-modulated RT (IMRT) first, and volumetric modulated arc therapy (VMAT) subsequently. In fact, these techniques allow a higher dose conformity due to the steeper dose gradients around the target volume, reduced irradiation of organs at risk (OAR), and therefore the delivery of higher RT doses to the tumor [9-13]. A meta-analysis showed that IMRT, compared to 3D-CRT, can achieve lower G2-4 rectal toxicity rates and improve biochemical relapse-free survival [14].

However, it is well known that modulated RT techniques lead to low-level doses in larger body volumes compared to 3D-CRT. Theoretically, this characteristic could increase the risk of RT-induced carcinogenesis and then of second tumors. The theoretically increased risk of IMRT/VMAT induced second tumors in PCa patients has been largely discussed in literature. Several studies addressed this topic mainly in planning and dosimetric analyses [15-18]. However, comparisons between 3D-CRT and modulated RT techniques in terms of second tumors incidence based on real clinical data are still lacking.

Therefore, the aim of this retrospective analysis was to evaluate the impact of RT technique (3D-CRT vs IMRT/ VMAT) on the incidence of second primary tumors in PCa patients. Moreover, also the impact of ADT and irradiated volumes in terms of delivery or not of prophylactic nodal irradiation (PNI), was investigated.

\section{Methods}

\section{End points and study design}

The primary end point of this study was the correlation of RT technique with second primary cancers incidence in PCa. The secondary objectives of the analysis were the correlation of ADT and PNI on the same outcome. The study design was a monocentric retrospective analysis on all PCa patients previously treated with external beam RT (EBRT) included in our institutional PCa database.

\section{Inclusion criteria}

Inclusion criteria were as follows: 1) histologically confirmed prostatic adenocarcinoma; 2) curative aim of RT; 3 ) age $>18$ years. Exclusion criteria were: 1) patients with distant metastases; 2) palliative aim of RT; 3) previous chemotherapy or RT on any site of the body; 4) some diseases potentially affecting tolerance to radiation therapy and potentially associated to a higher risk of cancer: ulcerative colitis, Crohn's disease, familial adenomatous polyposis, and bladder papilloma; 5) patients with malignancies diagnosed prior to $\mathrm{PCa}$ diagnosis; 6) patients with malignancies diagnosed during $\mathrm{PCa}$ staging and planning.

\section{Radiotherapy}

All patients underwent computed tomography (CT) simulation in supine position using a personalized immobilization system. In some patients, Positron Emission Tomography (18F-choline or $11 \mathrm{C}$-choline or $68-\mathrm{Ga}-$ PSMA) - CT simulation and/or CT-simulation image fusion with MRI scans were performed. The Clinical Target Volumes (CTV) were defined based on risk categories to include only the prostate (or prostatic bed) $+/-$ seminal vesicles or also pelvic lymph nodes. An isotropic margin ranging between 5 and $10 \mathrm{~mm}$ was added to the CTV to define the Planning Target Volumes. The photon beam energy was $10-15 \mathrm{MV}$ and $6 \mathrm{MV}$ in patients treated with 3D-CRT and IMRT/VMAT, respectively. As previously described, daily set-up verification was performed using an Electronic Portal Imaging Device in most patients [19]. Only in a small minority of patients treated after 2016, set-up and organ motion evaluation was performed using a cone-beam CT. Dose specification and prescription were performed based on the International Commission of Radiation Unit reports 62 and 83 for 3D-CRT and IMRT/ VMAT techniques, respectively [20, 21]. ADT was prescribed according to risk categories.

\section{Follow-up}

Patients were monitored weekly during RT. For a more precise estimate of acute toxicity, patients were evaluated at one month after the end of RT. Subsequently the patients were followed biannually for the first 5 years and annually thereafter. At each visit total PSA, blood count and urine test were requested. During each visit, the patients underwent rectal exploration and an interview on any symptoms that appeared in previous months. In the case of at least 2 episodes of rectal bleeding, the patients were referred to proctoscopy and, in cases of persistent bleeding, to total colonoscopy. In the case of microscopic or macroscopic hematuria, in the absence of clear signs of inflammation, patients were asked to undergo cystoscopy. In the case of recurrent bleeding, a renal and ureteral ultrasound was prescribed. Overall, 193 patients were lost at follow-up.

\section{Statistical analysis}

The IBM SPSS Version 22.0 software package was used for statistical computation (IBM Corp, Armonk, NY, USA). Survival estimates were calculated by the Kaplan-Meier product-limit method and compared with the log-rank test. Multivariate analysis was performed using a Cox regression model [22]. Also a Fine and Gray competing risk survival regression analysis was performed in $\mathrm{R}$ (version 3.5.2) using the library package "survival" and "cmprsk" to consider deaths from other causes as competing events. A $p<0.05$ value was considered statistically significant. The impact of RT technique (3D-CRT vs IMRT/VMAT), ADT (yes or not), and PNI (yes or not) on the incidence of second 
primary tumors was estimated. Second tumors incidence was evaluated not only as "any second tumor" detected during the follow-up but also considering other 2 groups: i) second tumors in the pelvis and ii) second tumors in the abdomen or pelvis. In cases of doubtful interpretation of the information contained in the database for the purposes of this stratification, the diagnostic images of the second tumor were analysed.

\section{Ethical issues}

The local institutional review board approved this analysis (311/2019/Oss/AOUBo, ICAROS-1 study). Only patients who had provided a written informed consent to the scientific use of their data were included.

\section{Results}

\section{Patients characteristics}

We included in the analysis $2526 \mathrm{PCa}$ patients who met the inclusion criteria and received EBRT between 2002 and 2018. Median follow-up was 72 months (range: 9185 months) and median age was 71 years (range: 43-93 years). The RT settings were definitive (54.2\%), adjuvant
(32.8\%), or salvage treatment (13.0\%). Patients were treated with 3D-CRT technique (21.3\%), IMRT (68.1\%), or VMAT (10.6\%). Total 3D-CRT median delivered dose was $70 \mathrm{~Gy}$ (median dose/fraction: $2.5 \mathrm{~Gy}$ ) and the total IMRT/VMAT median dose was 67.5 Gy (median dose/ fraction: $2.6 \mathrm{~Gy}$ ). PNI and ADT were prescribed to 1294 (51.2\%) and 1689 (66.9\%) patients, respectively. Patients treated with 3D-CRT and IMRT/VMAT received PNI in 39.4 and $54.4 \%$ of cases, respectively.

\section{Incidence of second tumors}

Ninety-two (3.6\%) cases of second tumors were recorded. Median interval between RT and second tumor was 48 months (range: 9-152 months) and median age was 70 years (range: $45-83$ years) at diagnosis of the second cancer. Moreover, there were 31 (1.2\%), 26 (1.0\%), and $35(1.4 \%)$ cases of second primary cancers detected in the pelvis, abdomen, and other sites, respectively. Considering the group of younger patients $(\leq 66$ years: first quartile), we recorded $25 \mathrm{~s}$ tumors out of 688 cases. This information on second tumors was collected from patient chart-records. Table 1 shows the number and

Table 1 Number and crude percentages of detected second tumors

\begin{tabular}{|c|c|c|c|c|}
\hline \multirow{2}{*}{$\begin{array}{l}\text { Incidence of second } \\
\text { tumors }\end{array}$} & \multicolumn{3}{|l|}{ Technique } & \multirow{2}{*}{$\begin{array}{l}\text { Total } \\
2526(\%)\end{array}$} \\
\hline & $\begin{array}{l}\text { 3D-CRT } \\
538(\%)\end{array}$ & $\begin{array}{l}\text { IMRT } \\
1719(\%)\end{array}$ & $\begin{array}{l}\text { VMAT } \\
269(\%)\end{array}$ & \\
\hline No & $515(95.7)$ & 1660 (96.6) & $259(96.3)$ & $2434(96.4)$ \\
\hline \multicolumn{5}{|l|}{ Pelvis } \\
\hline Bladder & $8(1.5)$ & $19(1.1)$ & $4(1.5)$ & $31(1.2)$ \\
\hline Rectum & $0(0.0)$ & $4(0.2)$ & $1(0.4)$ & $5(0.2)$ \\
\hline Sigma & $0(0.0)$ & $2(0.1)$ & $0(0.0)$ & $2(0.1)$ \\
\hline \multicolumn{5}{|l|}{ Abdomen } \\
\hline Colon & $0(0.0)$ & $4(0.2)$ & $1(0.4)$ & $5(0.2)$ \\
\hline Stomach & $0(0.0)$ & $4(0.2)$ & $1(0.4)$ & $5(0.2)$ \\
\hline Kidney & $2(0.4)$ & $1(0.1)$ & $0(0.0)$ & $3(0.1)$ \\
\hline Pancreas & $2(0.4)$ & $0(0.0)$ & $0(0.0)$ & $2(0.1)$ \\
\hline Small bowel (duodenal) & $1(0.2)$ & $0(0.0)$ & $0(0.0)$ & $1(0.0)$ \\
\hline Small bowel (ileum) & $0(0.0)$ & $1(0.1)$ & $0(0.0)$ & $1(0.0)$ \\
\hline \multicolumn{5}{|l|}{ Other sites } \\
\hline Lung & $4(0.7)$ & $6(0.3)$ & $2(0.7)$ & $12(0.5)$ \\
\hline Melanoma & $1(0.2)$ & $7(0.4)$ & $0(0.0)$ & $8(0.3)$ \\
\hline Skin & $0(0.0)$ & $6(0.3)$ & $0(0.0)$ & $6(0.2)$ \\
\hline Head and neck & $2(0.4)$ & $2(0.1)$ & $0(0.0)$ & $4(0.2)$ \\
\hline Brain & $0(0.0)$ & $3(0.2)$ & $0(0.0)$ & $3(0.1)$ \\
\hline Lymphoma & $0(0.0)$ & $0(0.0)$ & $1(0.4)$ & $1(0.0)$ \\
\hline Leukaemia & $1(0.0)$ & $0(0.0)$ & $0(0.0)$ & $1(0.0)$ \\
\hline Oesophagus & $1(0.2)$ & $0(0.0)$ & $0(0.0)$ & $1(0.0)$ \\
\hline Lip & $1(0.2)$ & $0(0.0)$ & $0(0.0)$ & $1(0.0)$ \\
\hline
\end{tabular}

Legend: 3D-CRT three-dimensional conformal radiotherapy; IMRT Intensity modulated radiotherapy, VMAT volumetric modulated radiotherapy 
percentages of detected second tumors. The 10-year actuarial cumulative incidence of second tumors was $14.4 \%$.

\section{Impact of treatment characteristics on second tumors incidence}

For the entire cohort, the calculated 10-year second tumorfree survival (STFS) in patients treated with 3D-CRT and IMRT/VMAT was 85.8 and $84.5 \%$, respectively ( $p: .627$ ).

At univariate analysis, 10-year STFS in patients treated with or without PNI was 84.9 and $88.1 \%$, respectively ( $p$ : .770). Ten-year STFS in patients receiving or not ADT was 83.8 and $92.8 \%$, respectively (p: .999). A significantly higher 10-year cumulative incidence of second tumors in the pelvis was registered in patients treated with IMRT/VMAT compared to 3D-CRT $(10.7 \%$ vs $6.0 \%$; $p$ : .033). Moreover, PNI showed a trend $(p: 0.1)$ for increased 10-year incidence of second tumors in both pelvis $(9.4 \%$ vs $5.6 \%, p: .092)$ and pelvis-abdomen $(10.9 \%$ vs 7.4\%, p: .064) (Table 2, Fig. 1).

Stratifying patients in 4 groups according to used RT technique and irradiated volumes, a statistically significant difference was recorded in terms of STFS in the pelvis $(p: .044)$. The 10-year STFS were as follows: 3DCRT without PNI: 96.6\%; 3D-CRT with PNI: 93.7\%; IMRT/VMAT without PNI: 89.9\%; and IMRT/VMAT with PNI: 87.6\% (Table 2, Fig. 2).

On multivariate analysis (Table 3), the lower incidence of second pelvic cancers in patients treated with $3 \mathrm{D}$ CRT was confirmed (hazard ratio [HR]: 2.42, 95\%CI: 1.07-5.47, $p:$.034). Furthermore, the incidence of second pelvis-abdomen cancers were found to have a trend in case of PNI delivery (HR: 1.63, 95\%CI: 0.95-2.79, $p$ : .067). Moreover, in a separate multivariate analysis where RT techniques and irradiated volumes were combined, patients treated with IMRT/VMAT plus PNI were found to have a significantly increased risk of second pelvic cancers (HR: 3.24, 95\%CI: 1.09-9.65, p: .035) and second pelvis-abdomen cancers (HR: 2.61, 95\%CI: $1.06-$ $6.41, p: .037)$. The Fine and Gray regression model analysis confirmed a trend of influence of IMRT/VMAT on the incidence of pelvic tumors (p: 0.058). Furthermore, considering the combination of radiotherapy technique and irradiated volumes, the same model confirmed the significant impact of IMRT/VMAT plus PNI on pelvic (p: 0.033) and pelvic or abdominal (p: 0.034) second tumors, using 3D-CRT without PNI as a reference.

\section{Discussion}

We performed an analysis on the incidence of second cancers in PCa patients treated with EBRT to evaluate the impact of RT technique, irradiated volumes, and ADT. The analysis showed a significant correlation between 10-year incidence of second tumors located in the pelvis and RT technique [3D-CRT vs IMRT (6.0\% vs $10.7 \%, p$ : .033)], while PNI showed a trend for increased 10-year incidence of second tumors in both pelvis (9.4\% vs 5.6\%, p. .092) and pelvis-abdomen (10.9\% vs 7.4\%, p: .064).

Our study has several limitations which simply make it a hypothesis generating analysis. First, the median follow-up is relatively short (72 months). In fact, in a cohort of Hodgkin's Lymphoma patients treated with RT, the median latency time to second tumor was 7.5 years [23]. We cannot completely exclude that with a longer follow-up, the recorded differences could lose their statistical significance. Furthermore, not having defined a minimum follow-up duration as an inclusion criterion, it is possible that some of the second malignancies

Table 2 Univariate analysis (10-year Second Tumor-Free Survival)

\begin{tabular}{|c|c|c|c|c|c|c|c|c|}
\hline \multirow[t]{2}{*}{ Variables } & & \multirow{2}{*}{$\begin{array}{l}\text { No. of patients } \\
(\%)\end{array}$} & \multicolumn{2}{|c|}{ all sites } & \multicolumn{2}{|c|}{ pelvis } & \multicolumn{2}{|c|}{ pelvis/abdomen } \\
\hline & & & STFS & $p$ & STFS & $p$ & STFS & $p$ \\
\hline \multirow[t]{2}{*}{ Radiotherapy technique } & 3D-CRT & $538(21.3)$ & 85.8 & .627 & 94.0 & .033 & 92.2 & .125 \\
\hline & IMRTNMAT & $1988(78.7)$ & 84.5 & & 89.3 & & 87.5 & \\
\hline \multirow[t]{2}{*}{ Prophylactic nodal irradiation } & no & $1232(48.8)$ & 88.1 & .770 & 94.4 & .092 & 92.6 & .064 \\
\hline & yes & $1294(51.2)$ & 84.9 & & 90.6 & & 89.1 & \\
\hline \multirow[t]{2}{*}{ Androgen deprivation therapy } & no & 837 (33.1) & 92.8 & .999 & 93.1 & .546 & 92.0 & .345 \\
\hline & yes & $1689(66.9)$ & 83.8 & & 91.9 & & 89.9 & \\
\hline \multirow[t]{2}{*}{ Age, years } & $\leq 66$ & $688(27.2)$ & 85.3 & .352 & 90.5 & .981 & 89.6 & .374 \\
\hline & $>66$ & $1838(72.8)$ & 86.0 & & 93.5 & & 91.4 & \\
\hline \multirow{4}{*}{$\begin{array}{l}\text { Combination of radiotherapy } \\
\text { technique and irradiated volumes }\end{array}$} & 3D-CRT without PNI & $326(12.9)$ & 91.0 & .887 & 96.6 & .044 & 95.3 & .140 \\
\hline & IMRTNMAT without PNI & 906 (35.9) & 78.9 & & 89.9 & & 87.8 & \\
\hline & 3D-CRT with PNI & $212(8.4)$ & 85.6 & & 93.7 & & 91.1 & \\
\hline & IMRTNMAT with PNI & $1082(42.8)$ & 86.6 & & 87.6 & & 86.3 & \\
\hline
\end{tabular}

Legend: 3D-CRT three-dimensional conformal radiotherapy, IMRT Intensity modulated radiotherapy, PNI prophylactic nodal irradiation, STFS second tumor free survival, VMAT volumetric modulated radiotherapy 


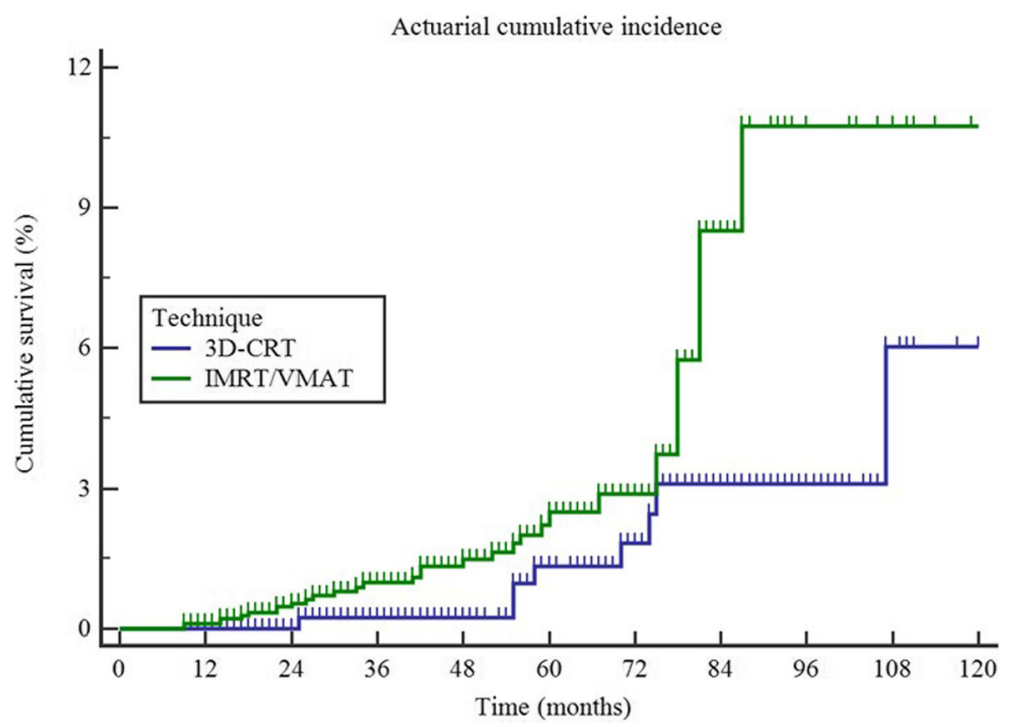

Number at risk

$3 \mathrm{D}-\mathrm{CRT}$

$\begin{array}{ccccccccccc}538 & 537 & 495 & 449 & 364 & 332 & 247 & 174 & 114 & 86 & 71 \\ \text { IMRT/VMAT } & & & & & & & & & & \\ 1988 & 1978 & 1836 & 1570 & 1230 & 954 & 550 & 330 & 236 & 123 & 67\end{array}$

Fig. 1 actuarial cumulative risk of pelvic second primary tumors after radiotherapy (3D-conformal therapy vs modulated techniques; p: .033)

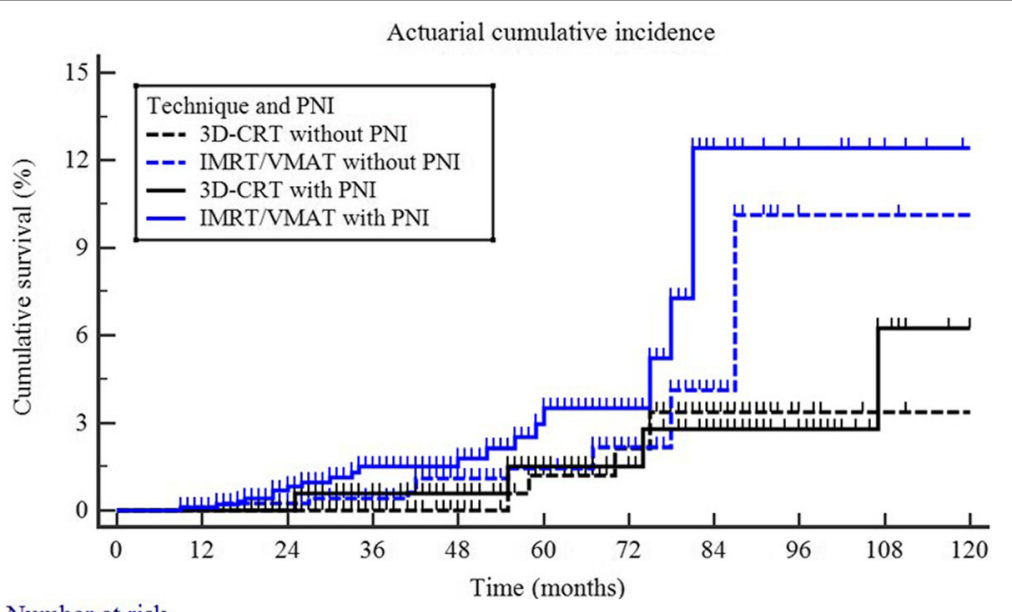

Number at risk

3D-CRT without PNI

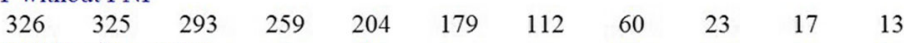

IMRT/VMAT without PNI

$\begin{array}{lllllllllll}906 & 900 & 823 & 667 & 498 & 362 & 141 & 51 & 35 & 30 & 18\end{array}$

3D-CRT with PNI

$\begin{array}{lllllllllll}212 & 212 & 202 & 190 & 160 & 153 & 135 & 114 & 91 & 69 & 58\end{array}$

IMRT/VMAT with PNI

$\begin{array}{lllllllllll}1082 & 1078 & 1013 & 903 & 732 & 592 & 409 & 279 & 201 & 93 & 49\end{array}$

Fig. 2 actuarial cumulative risk of pelvic second primary tumors after radiotherapy (3D-conformal radiotherapy without prophylactic nodal irradiation versus 3D-conformal radiotherapy with prophylactic nodal irradiation versus modulated radiotherapy techniques without prophylactic nodal irradiation versus modulated radiotherapy techniques with prophylactic nodal irradiation; p: .044) 
Table 3 Multivariate analysis on second tumor free survival

\begin{tabular}{|c|c|c|c|c|c|c|c|}
\hline \multirow[t]{2}{*}{ Variable } & \multirow[t]{2}{*}{ value } & \multicolumn{2}{|c|}{ pelvic } & \multicolumn{4}{|c|}{ Pelvic-abdominal } \\
\hline & & $H R$ & $95 \% \mathrm{Cl}$ & $p$ & $H R$ & $95 \% \mathrm{Cl}$ & $p$ \\
\hline \multirow[t]{2}{*}{ Radiotherapy technique } & 3D-CRT & Ref & & & & & \\
\hline & IMRTNMMAT & 2.42 & $1.07-5.47$ & .034 & & & \\
\hline \multirow[t]{2}{*}{ Prophylactic nodal irradiation } & No & & & & Ref & & \\
\hline & Yes & & & & 1.63 & $0.95-2.79$ & .067 \\
\hline \multirow{4}{*}{$\begin{array}{l}\text { Combination of radiotherapy } \\
\text { technique and irradiated volumes }\end{array}$} & 3D-CRT without PNI & Ref & & & Ref & & \\
\hline & IMRTNMAT without PNI & 1.70 & $0.53-5.51$ & .375 & 1.66 & $0.64-4.29$ & .294 \\
\hline & 3D-CRT with PNI & 1.10 & $0.27-4.46$ & .892 & 1.73 & $0.61-4.92$ & .303 \\
\hline & IMRTNMAT with PNI & 3.24 & $1.09-9.65$ & .035 & 2.61 & $1.06-6.41$ & .037 \\
\hline
\end{tabular}

Legend: 3D-CRT three-dimensional conformal radiotherapy, IMRT Intensity modulated radiotherapy, PNI prophylactic nodal irradiation, VMAT volumetric modulated radiotherapy

diagnosed during the follow-up had arisen before RT. This aspect may have influenced the results of the analysis. Moreover, the sample size (2526 patients) can be considered relatively small. In fact, other studies in this field $[3,6,24]$, two of which were registry studies [3, 24], included 9538-619,479 patients. Even this small sample size could potentially have affected the results of the analysis. Furthermore, although image guided RT could add a non-negligible risk for second tumors when daily set-up verification with high-resolution modality is performed [13], we did not consider this issue in our analysis. However, it should be noted that no extra dose was delivered for set-up verification in most patients. Furthermore, only a small minority of patients treated in the last 2 years had their treatment position and organ motion checked using a daily cone-beam CT. In addition, the evaluation of other potential factors correlated with second tumors are lacking in our analysis. For example, the first 2 primary tumors recorded in this study were bladder and lung cancers and both are smoking-related malignancies. Therefore, it would have been interesting to evaluate the impact of RT techniques also considering the smoking habits of individual patients. Unfortunately, even in this case, this data is only available in a minority of patients and therefore could not be analysed. Moreover, it should be noted that until 2009 (when all patients of the 3D-CRT group were treated), the cranial margin for pelvic nodes delineation was at the level of the anterior margin of the S1 vertebra. From 2009 (when all patients were treated with modulated techniques), this margin was moved slightly cranially, at the level of the S1-L5 interspace. Therefore, we cannot rule out that most patients undergoing modulated techniques received a slightly more extensive irradiation in the cranial direction and that this may have influenced the results of our analysis.

Finally, patients with short observation time were not excluded in order to consider a reasonable latency time between RT and onset of the second tumor. For example, in a previous study, the analysis of second solid cancers was based only on 5-year survivors and analysis of leukemia were based only on 2-year survivors [25]. However, given the uncertainty about the latency times of second tumors occurrence, we decided to use a conservative criterion and therefore to include all primitive tumors diagnosed after RT.

In the past, even if the results are somehow contradictory [26] and the incidence of second tumors could also be attributed to age and lifestyles [6], several analyses showed an increased risk for second tumors after EBRT of PCa [3-5, 24]. Probably these data should be considered with caution. In fact, in previously cited studies [3-5, 24], the incidence of second tumors was evaluated by comparing PCa patients who underwent RT with subjects receiving other treatments, mainly represented by radical prostatectomy (RP). In this regard, it should be noted that in different risk categories, RT and RP are considered as alternative therapeutic options. However, in daily clinical practice, the choice between the two treatments is often based on patient's comorbidities. In particular, RT is preferred to RP in case of contraindications to surgery. These contraindications (COPD, cardiovascular diseases, metabolic syndrome) are more frequent in smoking patients and these subjects are obviously more prone to smoke-related malignancies such as bladder or lung tumors.

Some studies evaluated also the impact of RT technique on the incidence of second tumors. In particular, three meta-analyses uniformly recorded a higher incidence of second rectal tumors after EBRT but not after brachytherapy $[4,5,26]$. In another study no differences were observed in terms of overall incidence of second tumors between 2D-conventional and 3D-CRT but only an advantage in patients undergoing 3D-CRT in terms of second rectal tumors. In the same analysis, no significant differences were observed based on beams photons energy (> $10 \mathrm{MV}$ versus $\leq 10 \mathrm{MV}$ ) but a reduction in colon and leukaemia tumors in patients undergoing brachytherapy compared to those treated with external beams [25].

However, to the best of our knowledge, our study is the first analysis comparing 3D-CRT vs IMRT/VMAT 
techniques and evaluating also the impact of PNI and ADT. Furthermore, we considered the incidence of second tumors in different body regions (pelvis, pelvis or abdomen, and all together). The results of our analysis based on clinical data are in agreement with several dosimetric and planning studies predicting a higher incidence of bladder and/or rectal second cancers in patients treated with modulated techniques [15-18].

More generally, our study showed a $14.4 \%$ 10-year incidence of second tumors. Considering the favourable prognosis related to $\mathrm{PCa}$ (10-year OS: $87.3 \%$ in our series), this result should stimulate attention during the follow-up of patients not only to eventual PCa relapse but also to the risk of second tumors. In particular, haematuria or rectal bleeding should not be automatically considered as late RT induced toxicity but should also lead to further investigations on the possibility of bladder or rectal cancer, respectively.

Given the increased risk of radiation induced second tumors in $\mathrm{PCa}$ patients receiving $\mathrm{RT}$, this possibility should be discussed with patients before treatment [3]. Based on our analysis, not showing a significant increase in the overall incidence of second cancers, further explanations about the potential additional risk from modulated RT techniques seem not required.

However, further analysis with prolonged follow-up, possibly on larger patients' population and considering other risk factors such as smoking habits, should be performed to confirm our findings.

\section{Conclusions}

The results of our study suggest a correlation between the use of modulated techniques and the incidence of second pelvic tumors in RT of PCa. Furthermore, the combination of modulated techniques and PNI seems to increase the incidence of second abdominal-pelvic tumors. These results appear relevant given that: i) they justify the planning of further studies on this topic; ii) if confirmed by these future analyses, they would represent the first "clinical" confirmation of the hypothetical impact of modulated techniques on the incidence of second tumors; iii) these results suggest particular attention, in the follow up of patients treated with modulated techniques, to the early diagnosis of second pelvic tumors and, in case of PNI, also of second abdominal tumors.

\footnotetext{
Abbreviations

3D-CRT: 3-dimensional conformal RT; ADT: Androgen deprivation therapy; CT: Computed tomography; CTV: Clinical Target Volumes; EBRT: External beam RT; IMRT: Intensity-modulated RT; OAR: Organs at risk; PCa: Prostate cancer; PNI: Prophylactic nodal irradiation; RT: Radiotherapy; STFS: Second tumor free survival; VMAT: Volumetric modulated arc therapy
}

\section{Authors' contributions}

Conception and Design: MB, SiC and AGM; Data Collection: MB, ES, GM, SB, $M N, G S, A R A$ and FD; Analysis and Interpretation of Data: MB, SaC, LS, W and AGM; Manuscript Writing MB, ES, ARA, LS and AGM. All authors read and approved the final manuscript and gave consent to publication.

\section{Funding}

This research did not receive any specific grant from funding agencies in the public, commercial, or not-for-profit sectors.

\section{Availability of data and materials}

The datasets used and/or analysed during the current study are available from the corresponding author on reasonable request.

Ethics approval and consent to participate

This study was approved by the local institutional review board // comitato Etico di Area Vasta Emilia Centro della Regione Emilia-Romagna [CE-AVEC] (311/2019/Oss/AOUBo, ICAROS-1 study), and written informed consent was obtained from all the included patients.

Consent for publication

Not applicable.

\section{Competing interests}

no actual or potential conflicts of interest do exist regarding this paper.

\section{Author details}

${ }^{1}$ Radiation Oncology Center, Department of Experimental, Diagnostic and Specialty Medicine - DIMES, University of Bologna, S. Orsola-Malpighi Hospital, via Giuseppe Massarenti 9, 40138 Bologna, Italy. ${ }^{2}$ Radiotherapy Unit, Gemelli Molise Hospital, Catholic University of Sacred Heart, Campobasso, Italy. ${ }^{3}$ Medical Physic Unit, Gemelli Molise Hospital, Catholic University of Sacred Heart, Campobasso, Italy. ${ }^{4}$ Fondazione Policlinico Universitario "A. Gemelli" IRCCS, UOC Radioterapia Oncologica, Dipartimento di Diagnostica per Immagini, Radioterapia Oncologica ed Ematologia, Rome, Italy.

${ }^{5}$ Università Cattolica del Sacro Cuore, Istituto di Radiologia, Rome, Italy.

${ }^{6}$ Medical Physics Unit, "S. Orsola-Malpighi" Hospital, Bologna, Italy.

Received: 19 September 2019 Accepted: 27 January 2020

Published online: 03 February 2020

\section{References}

1. Siegel RL, Miller KD, Jemal A. Cancer statistics, 2019. CA Cancer J Clin. 2019; 69:7.

2. National Cancer Institute. SEER cancer statistics factsheets: anal cancer. http://seer.cancer.gov/statfacts/html/prost.html. Accessed June 29, 2019.

3. Aksnessæther BY, Lund JÅ, Myklebust TÅ, et al. Second cancers in radically treated Norwegian prostate cancer patients. Acta Oncol. 2019, 58:838-44. https://doi.org/10.1080/0284186X.2019.

4. Zhu Z, Zhao S, Liu Y, et al. Risk of secondary rectal cancer and colon cancer after radiotherapy for prostate cancer: a meta-analysis. Int J Color Dis. 2018; 33:1149-58. https://doi.org/10.1007/s00384-018-3114-7.

5. Wallis CJ, Mahar AL, Choo R, et al. Second malignancies after radiotherapy for prostate cancer: systematic review and meta-analysis. BMJ. 2016;352:1851. https://doi.org/10.1136/bmj.i851.

6. Hegemann NS, Schlesinger-Raab A, Ganswindt U, et al. Risk of second cancer following radiotherapy for prostate cancer: a population-based analysis. Radiat Oncol. 2017;12:2. https://doi.org/10.1186/s13014-016-0738-z.

7. Dearnaley DP, Khoo VS, Norman AR, et al. Comparison of radiation sideeffects of conformal and conventional radiotherapy in prostate cancer: a randomised trial. Lancet. 1999;353:267-272. PubMed PMID: 9929018.

8. Koper PC, Stroom JC, van Putten WL, et al. Acute morbidity reduction using 3DCRT for prostate carcinoma: a randomized study. Int J Radiat Oncol Biol Phys 1999:43:727-734. PubMed PMID: 10098427

9. National Comprehensive Cancer Network. Prostate cancer (version 2.2019). http://www.nccn.org/professionals/physician_gls/default.aspx. Accessed June 29, 2019

10. De Meerleer GO, Vakaet LA, De Gersem WR, De Wagter C, De Naeyer B, De Neve W. Radiotherapy of prostate cancer with or without intensity modulated beams: a planning comparison. Int J Radiat Oncol Biol Phys 2000;47:639-648. PubMed PMID: 10837946. 
11. Zelefsky MJ, Fuks Z, Hunt M, et al. High dose radiation delivered by intensity modulated conformal radiotherapy improves the outcome of localized prostate cancer. J Urol. 2001;166:876-881. Erratum in: J Urol. 2001;166:1839. PubMed PMID: 11490237.

12. Fenoglietto $P$, Laliberte $B$, Allaw $A$, et al. Persistently better treatment planning results of intensity-modulated (IMRT) over conformal radiotherapy (3D-CRT) in prostate cancer patients with significant variation of clinical target volume and/or organs-at-risk. Radiother Oncol. 2008;88:77-87. https:// doi.org/10.1016/j.radonc.2007.12.011.

13. Calandrino R, Perna L, Belli ML, et al. Second tumor induction risk in IMRT for prostate Cancer: an unbalanced comparison between surgery and radiotherapy? Health Phys. 2015;109:549-55. https://doi.org/10.1097/HP. 0000000000000362.

14. Yu T, Zhang $Q$, Zheng T, et al. The effectiveness of intensity modulated radiation therapy versus three-dimensional radiation therapy in prostate Cancer: a meta-analysis of the literatures. PLoS One. 2016;11:e0154499. https://doi.org/10.1371/journal.pone.0154499.

15. Kry SF, Salehpour M, Followill DS, et al. The calculated risk of fatal secondary malignancies from intensity-modulated radiation therapy. Int J Radiat Oncol Biol Phys 2005;62:1195-1203. PubMed PMID: 15990025.

16. Kry SF, Followill D, White RA, Stovall M, Kuban DA, Salehpour M. Uncertainty of calculated risk estimates for secondary malignancies after radiotherapy. Int J Radiat Oncol Biol Phys 2007;68:1265-1271. PubMed PMID: 17637398.

17. Stathakis S, Li J, Ma CC. Monte Carlo. Determination of radiation-induced cancer risks for prostate patients undergoing intensity- modulated radiation therapy. J Appl Clin Med Phys 2007:8:2685. PubMed PMID: 18449157.

18. Bednarz B, Athar $B, X u X G$. A comparative study on the risk of second primary cancers in out-of-field organs associated with radiotherapy of localized prostate carcinoma using Monte Carlo-based accelerator and patient models. Med Phys 2010;37:1987-1994. PubMed PMID: 20527532; PubMed Central PMCID: PMC2862056.

19. Deodato F, Cilla S, Massaccesi M, et al. Daily on-line set-up correction in 3Dconformal radiotherapy: is it feasible? Tumori. 2012;98:441-4.

20. International Commission on Radiation Units and Measurements. ICRU Report 62. Prescribing, recording, and reporting photon beam therapy (Supplement to ICRU Report 50). Bethesda, MD: ICRU; 1999.

21. International Commission on Radiation Unit and measurement. Journal of the ICRU 2010; 10, report 83. Oxford University Press.

22. Cox DR. Regression models and life tables. J R Stat Soc B. 1972;34:187-220.

23. Landgren O, Axdorph U, Fears TR, Porwit-MacDonald A, Wedelin C, Björkholm M. A population-based cohort study on early-stage Hodgkin lymphoma treated with radiotherapy alone: with special reference to older patients. Ann Oncol. 2006;17:1290-5.

24. Zhao S, Xie Q, Yang R, et al. High prevalence of secondary bladder cancer in men on radiotherapy for prostate cancer: evidence from a meta-analysis. Cancer Manag Res. 2019;11:587-98. https://doi.org/10.2147/CMAR.S185867.

25. Berrington de Gonzalez A, Wong J, Kleinerman R, Kim C, Morton L, Bekelman JE. Risk of second cancers according to radiation therapy technique and modality in prostate cancer survivors. Int J Radiat Oncol Biol Phys. 2015;91:295-302. https://doi.org/10.1016/j.j.jrobp.2014.10.040.

26. Lee YC, Hsieh CC, Li CY, Chuang JP, Lee JC. Secondary cancers after radiation therapy for primary prostate or rectal Cancer. World J Surg. 2016; 40:895-905. https://doi.org/10.1007/s00268-015-3324-x.

\section{Publisher's Note}

Springer Nature remains neutral with regard to jurisdictional claims in published maps and institutional affiliations.

Ready to submit your research? Choose BMC and benefit from:
- fast, convenient online submission
- thorough peer review by experienced researchers in your field
- rapid publication on acceptance
- support for research data, including large and complex data types
- gold Open Access which fosters wider collaboration and increased citations
- maximum visibility for your research: over 100M website views per year
At BMC, research is always in progress.
Learn more biomedcentral.com/submissions

